\title{
Caratterizzazione fenotipica di ceppi di Streptococcus agalactiae (streptococco di gruppo B) di origine umana e bovina isolati nel Nord Italia
}

\author{
Mattia Calzolari', Alessandra Polese², Paolo Bonilauri', Giuseppe Merialdi', Lidia Ricci², \\ Anna Nanetti ${ }^{3}$, Massimo Confalonieri', Michele Dottori'
}

'Istituto Zooprofilattico Sperimentale Lombardia ed Emilia Romagna, Sezione di Reggio Emilia

¿Laboratorio di Microbiologia A. O. "Santa Maria Nuova"; Reggio Emilia

${ }^{3}$ Unità Operativa di Microbiologia, Policlinico S. Orsola; Malpighi, Università degli Studi di Bologna (BO)

${ }^{4}$ Laboratorio di Microbiologia A. O. “G. da Saliceto"; Piacenza.

\section{Phenotypic characterization of strains of Streptococcus agalactiae (Group B Streptococci) of human and bovine origin isolated in Northern Italy.}

Key words: Streptococcus agalactiae, Group B Streptococci (GBS), serotype

\section{SUMMARY}

Serotype, haemolytic activity and CAMP reaction of 466 strains of Streptococcus agalactiae (269 of human origin and 197 of bovine origin) isolated in the North of Italy (Lombardia, Emilia) were evaluated. The presence of the rare serotype VII was recorded in 3 human strains and in I bovine isolates.

In human strains serotype III (32\%) was the most common followed by serotypes la (I7.I\%), V (I3.8\%), II (9.7\%), Ib (3.7\%), IV (3\%), VII (I.I\%); $19.6 \%$ of strains resulted nontypeable. Between bovine strains the most abundant was serotype IV (22.3\%), followed by Ib and III (5.6\%), II (5.I\%), la (3.6\%), V (3\%) and VII (0.5\%), a large number was nontypeable (54.3\%). Stains belonging to serotype of VI or VIII were not observed.

43 strains were anhemolytic (I5 of humane origin and 28 bovine origin) and 5 strains were CAMP-negative (2 humane and 3 bovine).

\section{INTRODUZIONE}

Streptococcus agalactiae, unica specie del gruppo $\mathrm{B}$ di Lancefield, può essere patogeno per diverse specie animali e per l'uomo. Questo batterio è uno dei principali agenti eziologici della mastite bovina, che provoca gravi perdite di reddito negli allevamenti da latte (1). Colonizza nell'uomo i distretti periferici dell'apparato digerente ed urogenitale senza essere solitamente patogeno, può però divenire causa di complicanze post partum ed infezioni delle vie urinarie (18). Molto importante è il ruolo che questo microrganismo ricopre nelle infezioni neonatali caratterizzate da sepsi e meningite, la colonizzazione del tratto genitale materno è associata alla colonizzazione del neonato e al rischio di infezione $(26,28,30)$. S. agalactiae può inoltre essere pericoloso per soggetti predisposti quali diabetici, malati di cancro ed HIV, nei quali può provocare batteriemie, endocarditi, osteomieliti, infezioni della pelle e dei tessuti molli (26).

Nonostante i ceppi di $S$. agalactiae di origine bovina e umana possano essere considerati due distinte popolazioni (13) è stato sperimentalmente dimostrato come ceppi di origine umana possano provocare mastite nei bovini (17). Inoltre sembrerebbe possibile la trasmissione orizzontale dai bovini a soggetti a rischio come gli allevatori (5). La caratterizzazione dei ceppi di differente origine può esserci d'aiuto per stabilire la similitudine e l'eventuale interscambio genetico esistente fra le due popolazioni.

Il tipo di emolisi è un carattere facilmente osservabile del quale abbiamo tenuto conto. S. agalactiae è solitamente in grado di produrre un'emolisina che lisa i globuli rossi su Agar sangue producendo $ß$-emolisi. Esistono però anche ceppi che non hanno questa capacità definiti y-emolitici (26). Abbiamo inoltre testato la capacità di questo microrganismo di completare l'emolisi prodotta da Staphilococcus aureus su Agar sangue (CAMP test). Nonostante questo sia uno dei test diagnostici più utilizzati per il riconoscimento di $S$. agalactiae sono stati descritti rari ceppi negativi a questa prova (15).

Un altro importante fattore caratteristico dei differenti ceppi di S. agalactiae sono i polisaccaridi capsulari, finora ne sono stati individuati 9 che permettono di distinguere altrettanti sierotipi: Ia, Ib, II, III, IV, V, VI, VII, VIII; presenti sia nei ceppi umani che bovini $(12,32)$.

I sierotipi Ia, Ib, II, III e V sono i più diffusi nell'uomo in Europa $(3,30,36)$, in Israele (23) e Nord dell'America $(14,25,33)$. In Giappone il VI 
e l'VIII sono i più comunemente isolati da donne gravide (18), i più frequenti da isolati ospedalieri in Senegal sono il II e il III (8) mentre in Kenya è stata riportata una certa abbondanza del Ia (22). Oltre alla regione geografica altri fattori influenzano le diffusioni dei diversi sierotipi, per esempio la provenienza etnica del soggetto da cui è stato isolato (16). Inoltre questa diffusione non è costante nel tempo (25), negli ultimi anni è stata riportata una forte emergenza del sierotipo $\mathrm{V}(3,9)$.

I ceppi bovini, isolati dal latte mastitico, presentano sempre un'alta percentuale di non tipizzabili rispetto agli umani (10), per quelli tipizzabili è stata rilevata una certa diffusione del sierotipo IV in Germania (4, 36), del II in Scozia (21) e in Indonesia (12), dell'Ia in Kenya (22) e del III in Canada $(10,20)$.

\section{MATERIALI E METODI}

Ceppi Batterici. I ceppi batterici di origine bovina sono stati isolati da latte mastitico (figura I) durante la normale attività diagnostica dell'Istituto Zooprofilattico Sperimentale della Lombardia e dell'Emilia Romagna dal febbraio 2001 al novembre 2002 e provengono dalle sezioni di Reggio Emilia (96 ceppi), Mantova (46 ceppi), Lodi (17 ceppi), Piacenza (11 ceppi), Brescia (10 ceppi), Parma (7 ceppi), Pavia (5 ceppi), Sondrio ( 5 ceppi). Per l'identificazione è stato utilizzato il CAMP test nella maggioranza dei casi e test al lattice (Phadebact ${ }^{\circledR}$ Strep B Test, Boule) per i microrganismi CAMP negativi che si sospettava potessero in ogni caso essere classificati come $S$. agalactiae.

I ceppi di origine umana sono stati isolati nel periodo fra il febbraio 2001 e il febbraio 2003 negli ospedali di Reggio Emilia (79 ceppi), Bologna (86 ceppi), Piacenza (83 ceppi) e Modena (21 ceppi). L'isolamento è stato effettuato da diversi distretti (figura I, tabella 1), la maggioranza proviene dall'apparato urogenitale (220), in particolare da tamponi vaginali (167 ceppi), urina (44 ceppi), liquido seminale (5 ceppi), tamponi uretrali (3 ceppi), liquido prostatico ( 1 ceppo); gli altri ceppi (49) sono stati isolati da ferite (18 ceppi), tamponi nasali e faringei (16 ceppi), sangue ( 6 ceppi), tamponi auricolari (4 ceppi), liquor (2 ceppi), liquido di drenaggio (2 ceppi), tamponi rettali (1 ceppo).

L'identificazione di questi ceppi è stato effettuato con test al lattice (Phadebact ${ }^{\mathbb{B}}$ Strep B Test, Boule) o tramite sistema automatizzato (Vitek ${ }^{\circledR}$ Plus, Biomerieux).

Sono stati testati 197 ceppi bovini e 269 ceppi umani per un totale di 466 ceppi. Questi stessi ceppi sono stati oggetto di approfondimenti inerenti la risposta a diversi antibiotici (6).
Sierotipizzazione, definizione emolisi, CAMP test. Dopo l'identificazione di specie i ceppi batterici sono stati congelati in appositi Microbank ${ }^{\mathrm{TM}}$ (ProLab Diagnostic) e conservati a $-80^{\circ} \mathrm{C}$. In seguito i ceppi da collezione così raccolti sono stati seminati su Agar sangue (Blod Agar base, Merck, con l'aggiunta del 5\% di sangue bovino), passaggio che ha permesso di rilevarne l'emolisi e di effettuare il CAMP test per quelli umani.

Successivamente abbiamo sierotipizzato tutti i ceppi con il kit fornito dalla ditta Denka-Seiken Co., Ltd.; per prima cosa abbiamo fatto crescere $\mathrm{i}$ microrganismi in Todd-Hewitt broth (Oxoid) a $30^{\circ} \mathrm{C}$ over-night, quindi siamo passati all'estrazione degli antigeni ottenuta prima digerendo con estratto pancreatico di suino e poi con trattamento termico a $120^{\circ} \mathrm{C}$ per 30 minuti. Gli estratti così ottenuti sono quindi stati testati tramite agglutinazione su vetrino, con antisieri monospecifici. Questi sono stati prodotti mediante estrazione da sieri di conigli iper-immunizzati verso ceppi batterici di $S$. agalactiae con antigeni tipo-specifici (Denka-Seiken Co., Ltd.).

Analisi statistiche. Per le analisi statistiche è stato utilizzato il test del $\chi^{2}$ (chi quadro), il limite di significatività è stato posto a $p<0.05$.

Tabella I. Provenienza dei ceppi di origine umana testati. In parentesi tonde () percentuali sul totale dei ceppi umani; contrassegnati con ${ }^{\mathrm{P}} \mathrm{i}$ ceppi isolati da materiale patologico

\begin{tabular}{lcc}
\hline FONTE DI ISOLAMENTO & UROGENITALI & ALTRO \\
\hline tamponi vaginali & $\mathrm{I} 67(62 . \mathrm{I})$ & \\
\hline urina & $44(\mathrm{I} 6.4)^{\mathrm{P}}$ & \\
\hline liquido seminale & $5(\mathrm{I} .9)^{\mathrm{P}}$ & \\
\hline tamponi uretrali & $3(\mathrm{I} . \mathrm{I})^{\mathrm{P}}$ & \\
\hline liquido prostatico & $\mathrm{I}(0.4)^{\mathrm{P}}$ & \\
\hline ferite & & $\mathrm{I}(6.7)^{\mathrm{P}}$ \\
\hline tamponi nasali e faringei & & $\mathrm{I}(5.9)^{\mathrm{P}}$ \\
\hline sangue & & $6(2.2)^{\mathrm{P}}$ \\
\hline tamponi auricolari & $4(1.5)$ \\
\hline liquor & & $2(0.7)^{\mathrm{P}}$ \\
\hline liquido di drenaggio & $2(0.7)^{\mathrm{P}}$ \\
\hline tamponi rettali & $\mathrm{I}(0.4)$ \\
\hline Totale: 269 & $220(8 \mathrm{I} .8)$ & $49(18.2)$ \\
\hline
\end{tabular}

\section{RISULTATI}

Per quanto riguarda i ceppi umani il sierotipo predominante è il III (32\%) seguito dal Ia $(17.1 \%)$, dal V (13.8\%), dal II (9.7\%), dal Ib (3.7\%), dal IV (3\%), e dal VII (1.1\%). Nessun ceppo appartiene al sierotipo VI e VIII e per un totale di 53 ceppi non è stato possibile identificare il sierotipo (non tipizzabili $16.3 \%$, autoagglutinanti $3.3 \%$; tabella 2).

Nei ceppi bovini il sierotipo IV è molto il più abbondante $(22.3 \%)$, seguito dal Ib e dal III $(5.6 \%)$, dal II (5.1\%), dal Ia (3.6\%), dal V (3\%) e dal VII $(0.5 \%)$. Non sono presenti ceppi dei sie- 
rotipi VI e VIII, inoltre si evidenzia un'alta percentuale di non tipizzabili (39.6\%), gli autoagglutinanti sono il $14.7 \%$ (tabella 2 ).

La distribuzione geografica dei sierotipi nei ceppi isolati da umani è omogenea fra le diverse province (tabella $2 ; \chi_{(24)}^{2}=32.58 p=0.113$ ). Esistono alcune eccezioni, le più evidenti riguardano il sierotipo $\mathrm{V}$, più abbondante a Bologna $(20.9 \%$ contro il $13.8 \%$ della popolazione umana testata; $\chi^{2}{ }_{(1)}=5.48$ $p=0.019)$ e il Ia più abbondante a Piacenza $(24.1 \%$ contro il $17.1 \%$ della popolazione umana testata; $\left.\chi_{(1)}^{2}=4.14 \mathrm{p}=0.042\right)$. Per gli isolati da bovini abbiamo ritenuto scorretto fare un confronto complessivo della distribuzione geografica dei sierotipi poiché i gruppi provenienti da alcune province sono esigui (tabella 2) e minerebbero l'attendibilità dei risultati. Possiamo però notare una certa similitudine fra le distribuzioni dei sierotipi nelle province di Reggio e Mantova, che presentano i gruppi di isolati più consistenti $\left(\chi_{(8)}^{2}=11.09 p=0.197\right)$. Un altro dato interessante, difficilmente attribuibile alla casualità, è l'abbondanza del sierotipo $\mathrm{Ib}$ a Piacenza (54.5\% contro il 5.6\% della popolazione bovina testata; $\left.\chi^{2}{ }_{(1)}=52.97 \mathrm{p}<0.01\right)$.

In totale sono stati individuati 43 ceppi non emolitici pari al $9.2 \%$ del totale, 15 sono di origine umana e 28 di origine bovina (tabella 3 ). Gli isolati di origine bovina mostrano una percentuale significativamente maggiore di ceppi non emolitici rispetto agli umani $\left(\chi_{(1)}^{2}=10.128 p<0.01\right)$ che spesso sono autoagglutinanti, non tipizzabili oppure, se tipizzabili, appartengono ai sierotipo $\mathrm{Ib}$ ( 2 ceppi), IV ( 2 ceppi) e III ( 1 ceppo). Per i ceppi umani solo il $5.6 \%$ non è emolitico. Molto interessante è notare come i ceppi umani $\gamma$-emolitici del III siano significativamente più abbondanti degli altri sierotipi (11 ceppi pari al $73.3 \%$ dei $\gamma$ umani; $\left.\chi^{2}(1)=12.80 p<0.01\right)$, gli altri isolati non emolitici appartengono al sierotipo Ia (1 ceppo) e V (1 ceppo) oppure non tipizzabili (1 ceppo) ed autoagglutinanti (1 ceppo).

I ceppi CAMP negativi sono in totale 5, 2 di origine umana appartenenti uno al sierotipo $\mathrm{V}$ e l'altro al Ia e 3 di origine bovina, tutti sierologicamente non tipizzabili.

\section{DISCUSSIONE}

Il dato di maggior interesse che emerge dai risultati è la presenza di ceppi del sierotipo VII (figura II, tabella 2), isolato raramente da campioni umani $(33,34,38)$ e non segnalato da altri autori nei bovini $(4,10,12,20,21,22,36)$. La virulenza dei microrganismi di questo sierotipo è stata studiata sui topi, nei quali possono provocare artrite per iniezione intravenosa (34). Il sierotipo VII è già stato rilevato con basse incidenze in altri paesi: in Canada nell'1\% degli isolati da soggetti umani adulti che avevano infezioni attribuibili a streptococchi di gruppo B (33) e in USA nello $0.7 \%$ di $S$. agalactiae isolati da neonati (38); in letteratura non abbiamo trovato nessuna segnalazione di questo sierotipo in Italia. Nel nostro caso i ceppi sono stati isolati da campioni di origine umana in 3 casi e in 1 caso da latte bovino, osservazione che ci permette di ipotizzare che questo sierotipo sia endemico nel nostro paese; tutti e $3 \mathrm{i}$ ceppi umani sono stati isolati dall'apparato urogenitale, 2 da tamponi vaginali e 1 da urina.

I microrganismi umani oggetto di studio sono stati isolati da reparti di microbiologia ospedaliera quindi comprendono sia ceppi non necessariamente patogeni, isolati da controlli di routine in soggetti sani (carriers), solitamente donne in stato di gravidanza, che isolati da pazienti nei quali provocavano effettivamente una patologia. Conoscendone la fonte d'origine possiamo distinguere queste due tipologie con buona approssimazione (tabella 1), grazie a questa suddivisione classifichiamo 172 ceppi $(63.9 \%)$ isolati da carriers e 97 ceppi $(36.1 \%)$ isolati da materiale patologico, quindi probabile causa d'infezioni.

La maggiore fonte di imprecisione in questo assunto è rappresentata dall'impossibilità di evidenziare, fra i ceppi isolati da tamponi vaginali, quelli che sono stati fonte di processi morbosi. Abbiamo in ogni caso ritenuto questa semplificazione accettabile perché recenti studi hanno dimostrato l'assenza di una correlazione causale diretta fra l'isolamento di streptococchi di gruppo B dalla vagina ed eventuali infezioni a carico di questo distretto (31).

La dinamica d'isolamento descritta influenza la distribuzione dei sierotipi poiché batteri con un determinato sierotipo sembrano avere un certo tropismo per particolari distretti (37), inoltre il tipo di capsula è correlato alla virulenza del ceppo (32). La prima osservazione sembrerebbe confermata anche dai nostri risultati siccome nell'apparato urogenitale $\mathrm{i}$ ceppi di sierotipo $\mathrm{V}$ sono più abbondanti che negli altri distretti (rispettivamente $15.4 \%, 34$ ceppi, contro $6.1 \%, 3$ ceppi), anche se questa differenza non è significativa $\left(\chi^{2}(1)=2.94\right.$ $p=0.086$ ). Tenendo conto della suddivisione presente in tabella 1 emerge inoltre come il sierotipo Ia sia più diffuso fra gli isolati da materiale patologico rispetto a quelli isolati da carriers (rispettivamente $21.7 \%, 22$ ceppi, contro il $13.4 \%, 23$ ceppi; $\chi_{(1)}^{2}=3.86 p=0.049$ ). Possiamo anche notare che i due ceppi provenienti da liquor appartengono al sierotipo III e $\mathrm{V}$, già indicati come $\mathrm{i}$ più frequenti nelle meningiti neonatali $(7,14,37)$.

I ceppi di origine bovina sono stati interamente isolati da latte mastitico ed osservando i dati emerge immediatamente l'alta percentuale di ceppi non 
Tabella 2. Sierotipi dei ceppi bovini e umani divisi per provincia di provenienza (Brescia-BS, Lodi-LO, Mantova-MN, Piacenza-PC, Parma- PR, Pavia-PV, Reggio Emilia-RE, Sondrio-SO, Bologna-BO, Modena-MO). In parentesi tonde ( ) percentuali relative per provincia; in parentesi quadre [] percentuali sul totale dei ceppi bovini e percentuali sul totale dei ceppi umani. Tot.b: totale ceppi bovini;Tot.u: totale ceppi umani; NT: non tipizzabili, A: autoagglutinanti.

\begin{tabular}{|c|c|c|c|c|c|c|c|c|c|c|c|c|c|c|}
\hline \multirow{2}{*}{ Sier. } & \multicolumn{8}{|c|}{ CEPPI BOVINI } & \multicolumn{6}{|c|}{ CEPPI UMANI } \\
\hline & BS & LO & $\mathrm{MN}$ & PC & $P R$ & PV & RE & SO & Tot.b & BO & MO & PC & RE & Tot.u \\
\hline a & $2(20)$ & $3(17.6)$ & $2(4.3)$ & & & & & & $7(3.6)$ & $13(15.1)$ & $4(19)$ & $20(24 . I)$ & $9(11.4)$ & $46(17.1)$ \\
\hline $\mathrm{lb}$ & $I(I 0)$ & I (5.9) & $2(4.3)$ & $6(54.4)$ & & & $\mathrm{I}(\mathrm{I})$ & & II (5.6) & $3(3.5)$ & $3(14.3)$ & $2(2.4)$ & $2(2.5)$ & $10(3.7)$ \\
\hline 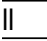 & & & $\mathrm{I}(2.2)$ & & & & $7(7.3)$ & $2(40)$ & $10(5.1)$ & $7(8.1)$ & $3(14.3)$ & $7(8.4)$ & $9(11.4)$ & $26(9.7)$ \\
\hline III & & $2(11.8$ & $2(4.3)$ & & $2(2$ & $\mathrm{I}(20)$ & $4(4.2)$ & & & 28 & $4(19)$ & $23(27.7)$ & 31 & \\
\hline IV & & & $15(32.6)$ & I (9.1) & & $2(40)$ & $25(26)$ & $I(20)$ & $44(22.3)$ & $3(3.5)$ & & $3(3.6)$ & $2(2.5)$ & $8(3)$ \\
\hline $\mathrm{V}$ & & $2(11.8)$ & $\mathrm{I}(2.2)$ & & & & $3(3.1)$ & & $6(3)$ & $18(20.9)$ & $2(9.5)$ & $8(9.6)$ & $9(I I .4)$ & $37(13.8)$ \\
\hline VII & & & I (2.2) & & & & & & I (0.5) & $\mathrm{I}(\mathrm{I} .2)$ & & $\mathrm{I}(\mathrm{I} .2)$ & $\mathrm{I}(1.3)$ & \\
\hline A & $3(30)$ & I (5.9) & $7(15.2)$ & I (9.I) & $2(28.6)$ & & $13(13.5)$ & $2(40)$ & 29 (I4.7) & & $3(14.3)$ & $3(3.6)$ & $3(3.8)$ & $9(3.3)$ \\
\hline NT & $4(40)$ & $8(47.1)$ & $15(32.6)$ & $3(27.3)$ & $3(42.9)$ & $2(40)$ & $43(44.8)$ & & 78 (39.6) & $13(15.1)$ & $2(9.5)$ & $16(19.3) 1$ & $13(16.5)$ & $44(16.3)$ \\
\hline Tot. & $10(5.1)$ & $17(8.6)$ & $46(23.4)$ & II (5.6) & $7(3.6)$ & $5(2.5)$ & $96(48.7)$ & $5(2.5)$ & 197 & $86(32)$ & $21(7.8)$ & $83(30.8)$ & $79(29.4)$ & 269 \\
\hline
\end{tabular}

Tabella 3. Distribuzione dei ceppi emolitici (ß) e non emolitici (y) nelle due popolazioni. Fra parentesi le percentuali relative riferite alle due popolazioni

\begin{tabular}{lcc}
\hline CEPPI & BOVINI & CEPPI UMANI \\
\hline emolisi $\beta$ & $169(85.8)$ & $254(94.4)$ \\
\hline emolisi $\gamma$ & $28(14.2)$ & $15(5.6)$ \\
\hline
\end{tabular}

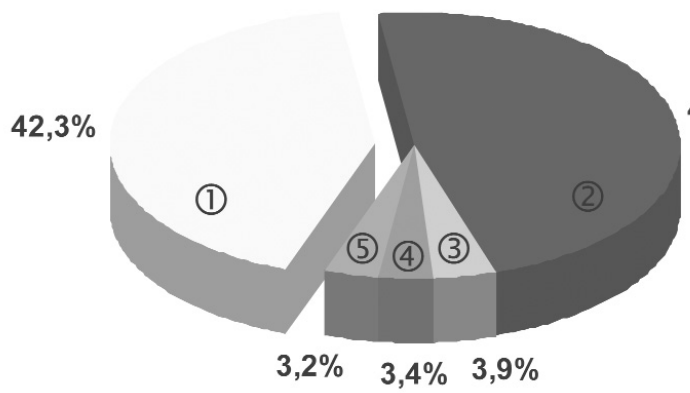

Figura I. Provenienza dei ceppi isolati. A sinistra, in bianco, $i$ ceppi bovini tutti isolati da latte (I); a destra, dal nero al grigio, $i$ ceppi umani isolati da: apparato urogenitale (2), ferite (3), tamponi faringei nasali (4), altro (5).

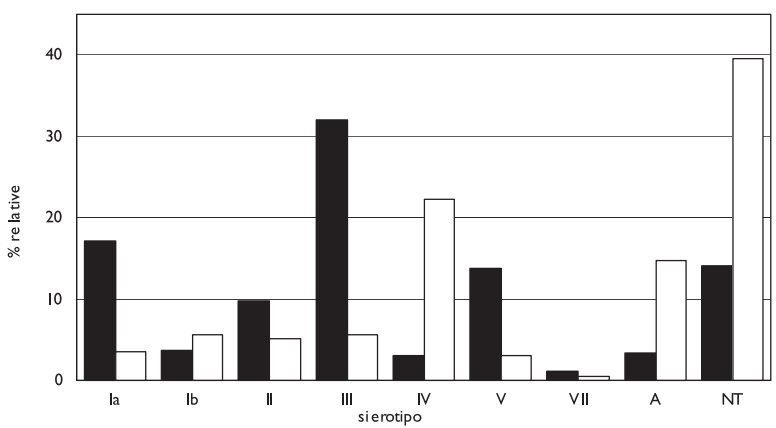

Figura II. Nei grafici è riportata la frequenza parziale (\%) dei sierotipi dei ceppi umani e bovini. In bianco sono riportati i ceppi di origine bovina $(\square)$ in nero quelli di origine umana $(\square)$.

tipizzabili (intorno al 40\%), possiamo formulare due ipotesi per spiegare questa situazione: la presenza di sierotipi non ancora identificati diffusi soprattutto fra i ceppi bovini, oppure la variazione del batterio dalla forma con capsula a quella senza (phase-shifth) dovuta alla permanenza nell'apparato mammario (10), fenomeno già descritto per ceppi coltivati in laboratorio $(27,29)$.

L'analisi dei dati ha permesso di confrontare la distribuzione dei sierotipi fra gli isolati umani e quelli bovini, consentendo di ricavare indicazioni sul possibile interscambio esistente fra $\mathrm{i}$ due. Questo confronto risulta ancora più indicativo perché la distribuzione geografica dei sierotipi è abbastanza omogenea. Il grafico presente in figura II mostra subito la grande lontananza esistente fra le distribuzioni dei sierotipi nelle due popolazioni $\left(\chi^{2}{ }_{(8)}=165.26 p<0.01\right)$, in particolare si evidenzia la predominanza del III (32\%) nell'uomo e del IV nei bovini $(22.3 \%)$, lontananza confermata anche dalla maggiore diffusione di ceppi $\gamma$-emolitici negli isolati da bovini rispetto agli isolati da umani (rispettivamente $14.2 \%$ e $5.6 \%$; tabella 3 ). Questa situazione indica uno scarso o inesistente rapporto fra ceppi umani e ceppi bovini. Effettivamente le normali norme profilattiche (es.: pastorizzazione del latte ad uso alimentare) dovrebbero escludere l'interscambio fra le due popolazioni, una simile considerazione trova conferma nella distribuzione dei sierotipi, molto simile fra ceppi bovini e umani, in paesi dove queste norme sono scarsamente rispettate $(5,22)$. Non va infine trascurata l'importanza che potrebbero avere questi dati nella formulazione di un eventuale vaccino, un approccio alternativo all'uso di antibiotici $(3,14,24,28)$ per la limitazione della diffusione delle patologie dovute a $S$. agalactiae, che sono in continuo aumento (35).

\section{RINGRAZIAMENTI}

Questi dati sono stati raccolti nell'ambito della ricerca ministeriale "Epidemiologia delle antibiotico resistenze di $S$. agalactiae di origine bovina e umana (PRF 2000202)" finanziata dal Ministero della Salute. Si ringrazia, per l'insostituibile collaborazione, tutto il personale dell'IZSLER, 
sezione di Reggio Emilia, in particolare Yuri Gherpelli.

\section{BIBLIOGRAFIA}

1. Ahl AS, Gibson CD, Kirk JH, Kaneene JB, Ahl JG. Cost of mastitis and its prevention in four dairy cattle herds on St Croix, US Virgin Islands. J Am Vet Med Assoc 1989; 194 (10): 1418-21.

2. Benson JA, Flores AE, Baker CJ, Hillier SL, Ferrieri P. Improved methods for typing nontypeable isolates of group B streptococci. Int J Med Microbiol 2002; 292 (1): 37-42.

3. Blumberg HM, Stephens DS, Modansky M, et al. Invasive group B streptococcal disease: the emergence of serotype V. J Infect Dis 1996 Feb;173 (2): 365-73.

4. Bopp V, Lammler C. Comparative studies on groupB streptococci isolated from bovine milk samples in Thuringia and Hesse. Zentralbl Veterinarmed [B] 1995; 42 (7): 427-33.

5. Brglez I, Stropnik Z, Batis J. Phagetypes of Human and Bovine Streptococcus agalactiae, isolate in Slovenia. Zbl Bakt Hyg B 1986; 182: 401-6.

6. Calzolari M, Polese A, Bonilauri P, Merialdi G, Ricci $\mathrm{L}$, Dottori M. Valutazione della sensibilità in vitro di ceppi di $S$. agalactiae di origine bovina e umana nei confronti di diversi antibiotici. Atti V Congresso Nazionale SIDiLV, 20-21 Novenbre 2003: 203-4.

7. Chatellier S, Ramanantsoa C, Harriau P, Rolland K, Rosenau A, Quentin R. Characterization of Streptococcus agalactiae strains by randomly amplified polymorphic DNA analysis. J Clin Microbiol 1997; 35 (10): 2573-9.

8. Cissè MF, Camara B, Diaw CF, Ba M. Serotypes and antibyotipes of Streptococcus agalactiae strains isolated in Dakar. Mèdecine et maladies infectieuses 2003, (33): 318-22.

9. Croak A, Abate G, Goodrum K, Modrzakowski M. Predominance of serotype $\mathrm{V}$ and frequency of erythromycin resistance in Streptococcus agalactiae in Ohio. Am J Obstet Gynecol 2003; 188 (5): 1148-50.

10. Daignault D, Guévremont E, Guillemette J-M, Messier S, Gottschalk M, Higgins R. Serotypes of Streptococcus agalactiae cultured from dairy milk samples in Québec. Can Vet J 2003; 44: 217-20.

11. Elliott JA, Farmer KD, Facklam RR. Sudden increase in isolation of Group B Streptococci, serotype $\mathrm{V}$, is not due to emergence of a new pulsedfield gel electrophoresis type. J Clin Microbiol 1998; 36 (7): 2115-6.

12. Estuningsih S, Soedarmanto I, Fink K, Lammler C, Wibawan IW. Studies on Streptococcus agalactiae isolated from bovine mastitis in Indonesia. J Vet Med B 2002; 49 (4): 185-7.

13. Finch LA, Martin DR. Human and bovine group B streptococci: two distinct populations. J Appl Bacteriol 1984 Oct; 57 (2): 273-8.

14. Harrison LH, Elliott JA, Dwyer DM, et al. Serotype distribution of invasive group B streptococcal isolates in Maryland: implications for vaccine formulation Maryland Emerging Infections Program. J Infect Dis 1998; 177 (4): 998-1002.

15. Hassan AA, Akineden O, Lammler C, HuberSchlenstedt R. Molecular characterization of phenotypically CAMP-negative Streptococcus agalactiae isolated from bovine mastitis. J Vet Med B Infect Dis
Vet Public Health 2002; 49 (5): 257-9.

16. Hickman ME, Rench MA, Ferrieri P, Baker CJ. Changing epidemiology of group B streptococcal colonization. Pediatrics 1999; 104 (2 Pt 1): 203-9.

17. Jensen NE. Experimental bovine group-B streptococcal mastitis induced by strains of human and bovine origin. Nord Vet Med Dec 1982; 34: 441-50.

18. La Placa M. Streptococchi ed enterococchi. In: Principi di microbiologia medica. Società editrice Esculapio 2001; IX ed: 235-6.

19. Lachenauer CS, Kasper DL, Shimada J, et al. Serotypes VI and VIII Predominate among Group B Streptococci Isolated from Pregnant Japanese Women. J Infect Dis 1999; 179: 1030-3.

20. Martinez G, Harel J, Higgins R, Lacouture S, Daignault D, Gottschalk M. Characterization of Streptococcus agalactiae isolates of bovine and human origin by randomly amplified polymorphic DNA analysis. J Clin Microbiol 2000; 38 (1): 71-8.

21. Morrison JRA, Wright CL. Streptococcus agalactiae serotypes in the south west of Scotland. Veterinary Record 1984; 115 (17): 439.

22. Mosabi JM, Arimi SM, Kang'ethe EK. Isolation and characterization of group B streptococci from human and bovine sources within and around Nairobi. Epidemiol Infect 1997; 118 (3): 215-20.

23. Nitzan Y (Zaidenzaig), Maayan M, Wajsman C. Streptococcus group B isolates in a regional hospital area. Med Microbiol Immunol 1980; 169: 21-30.

24. Paoletti LC, Kasper DL. Conjugate vaccines against group B Streptococcus types IV and VII. J Infect Dis 2002; 186 (1): 123-6.

25. Paoletti LJ, Bradford J, Paoletti LC. A Serotype VIII strain among colonizing group B Streptococcal isolates in Boston, Massachusetts. J Clin Microbiol 1999; 37 (11): 3759-60.

26. Ruoff KL, Whiley RA, Beighton D. Streptococcus. In: Manual of Clinical Microbiology. Murray PR, Baron EJ, Pfaller MA, Tenover FC, Yolken RH. American Society for Microbiology, Washington DC $7^{\text {th }}$ ed. 1999: 283-96.

27. Salasia SI, Wibawan IW, Lammler C, Sellin M. Phase variation in streptococci of serological group B. Characteristic properties of isolates from human and bovine infection. APMIS 1994; 102 (12): 925-30.

28. Schrag S, Gorwitz R, Fultz-Butts K, Schuchat A. Prevention of perinatal group $\mathrm{B}$ streptococcal disease. Revised guidelines from CDC. MMWR Recomm Rep 2002; 51 (11): 1-22.

29. Sellin M, Hakansson S, Norgren M. Phase-shift of polysaccharide capsule expression in group B streptococci, type III. Microb Pathog 1995; 18 (6): 401-15.

30. Sensini A, Tissi L, Verducci N, et al. Carriage of group B Streptococcus in pregnant women and newborns: a 2-year study at Perugia General Hospital. Clin Microbiol Infect. 1997; 3 (3): 324-8.

31. Shaw C, Mason M, Scoular A. Group B streptococcus carriage and vulvovaginal symptoms: causal or casual? A case-control study in a GUM clinic population. Sex Transm Infect 2003; 79 (3): 246-8.

32. Spellerberg B. Pathogenesis of neonatal Streptococcus agalactiae infections. Microbes and Infection 2000; 2: 1733-42.

33. Tyrrell GJ, Senzilet LD, Spika JS, et al. Invasive disease due to group B streptococcal infection in adults: results from a Canadian, population-based, active laboratory surveillance study-1996 Sentinel Health Unit Surveillance System Site Coordinators. J Infect Dis 2000; 182 (1): 168-73. 
34. von Hunolstein C, Parisi L, Tissi L, et al. Virulence properties of type VII Streptococcus agalactiae (group B streptococci) and immunochemical analysis of capsular type polysaccharide. J Med Microbiol 1999; 48 (11): 983-90.

35. Wessels MR, Kasper DL. The changing spectrum of group B streptococcal disease. N Engl J Med 1993; 328 (25): 1843-4.

36. Wibawan IWT, Lautrou Y, Lammler CH. Antibiotic resistance patterns and pigment production of
Streptococci of serological group B isolated from bovines and humans. J Vet Med 1991; 38: 731-6.

37. Wilkinson HW. Analysis of group B streptococcal types associated with disease in human infants and adults. J Clin Microbiol 1978; 7 (2): 176-9.

38. Zaleznik DF, Rench MA, Hillier S, et al. Invasive disease due to group B Streptococcus in pregnant women and neonates from diverse population groups. Clin Infect Dis 2000; 30 (2): 276-81.

\author{
Mattia Calzolari \\ Istituto Zooprofilattico Sperimentale della \\ Lombardia e dell'Emilia Romagna \\ Sezione Reggio Emilia \\ Via Pitagora, 2 \\ 42100 Reggio Emilia \\ Tel. 0522 921733; Fax 0522518639 \\ E-mail: matca@libero.it
}

